Article

\title{
The Tubby Torus as a Quotient Group
}

\author{
Sidney A. Morris ${ }^{1,2}$ (1)
}

1 School of Science, Engineering and Information Technology, Federation University Australia, P.O.B. 663, Ballarat, VIC 3353, Australia; morris.sidney@gmail.com

2 Department of Mathematics and Statistics, La Trobe University, Melbourne, VIC 3086, Australia

Received: 23 December 2019; Accepted: 17 January 2020; Published: 20 January 2020

check for updates

\begin{abstract}
Let $E$ be any metrizable nuclear locally convex space and $\widehat{E}$ the Pontryagin dual group of $E$. Then the topological group $\widehat{E}$ has the tubby torus (that is, the countably infinite product of copies of the circle group) as a quotient group if and only if $E$ does not have the weak topology. This extends results in the literature related to the Banach-Mazur separable quotient problem.
\end{abstract}

Keywords: torus; tubby torus; separable quotient problem; locally convex space; nuclear space; Banach space; pontryagin duality; weak topology

\section{Introduction and Preliminaries}

The Separable Quotient problem for Banach spaces has its roots in the 1930s and is due to Stefan Banach and Stanisław Mazur. While a positive answer is known for various classes of Banach spaces [1], such as reflexive Banach spaces, weakly compactly generated Banach spaces, and more generally Banach-like spaces [2], the general problem remains unsolved.

Problem 1. (Separable quotient problem for Banach spaces) Does every infinite-dimensional Banach space have a quotient Banach space which is separable and infinite-dimensional?

The following problem stated in [3] is also unsolved, but a negative answer to it would give a negative answer to Problem 1.

Problem 2. Does every infinite-dimensional Banach space have a quotient topological group which is homeomorphic to the countably infinite product, $\mathbb{R}^{\omega}$, of copies of $\mathbb{R}$ ?

This suggests another question which we have not seen mentioned in the literature. We state the problem and answer it.

Question 1. Does every infinite-dimensional Banach space have a quotient topological space which is homeomorphic to $\mathbb{R}^{\omega}$ ?

Question 1 has a positive answer, although it uses very powerful machinery due to Toruńczyk. It is known [4] that every infinite-dimensional Fréchet space $F$ (that is, a complete metrizable locally convex space) is homeomorphic to an infinite-dimensional Hilbert space $H$. So an infinite-dimensional Banach space $B$ (indeed an infinite-dimensional Fréchet space) is homeomorphic to an infinite-dimensional Hilbert space $H$, which obviously has the infinite-dimensional separable Hilbert space $\ell_{2}$ as a quotient. Further, by the separable case of Torunczyk's theorem which is known as the Kadec-Anderson theorem, the separable Fréchet space $\mathbb{R}^{\omega}$ is homeomorphic to $\ell_{2}$, from which the positive answer to Question 1 follows. 
Noting that Problem 2 remains open, it is natural to ask if every infinite-dimensional Banach space has a quotient topological group which is a separable metrizable topological group which is infinite-dimensional as a topological space. This was answered in the positive by the following theorem.

Theorem 1. [5] Every locally convex space E, which has a subspace which is an infinite-dimensional Fréchet space, has the tubby torus, $\mathbb{T}^{\omega}$, as a quotient group, where $\mathbb{T}$ is the compact circle group. In particular, this is the case if $E$ is an infinite-dimensional Banach space.

We should mention the following result.

Theorem 2. [6] If $E$ is any infinite-dimensional Fréchet space which is not a Banach space, then E has the locally convex space $\mathbb{R}^{\omega}$ as a quotient vector space.

Corollary 1. If $E$ is any infinite-dimensional Fréchet space which is not a Banach space, then E has the tubby torus $\mathbb{T}^{\omega}$ as a quotient group.

One might suspect that every infinite-dimensional locally convex space has the tubby torus as a quotient group. This is shown to be false in [5] for the free locally convex space $\varphi$ on a countably infinite discrete space. Indeed in [7] it is shown that if $X$ is a countably infinite $k_{\omega}$-space, then the free topological vector space on $X$, which is a connected infinite-dimensional (in the topological sense) topological group, does not have the tubby torus as a quotient group or even any infinite-dimensional (in the topological sense) metrizable quotient group.

It was recently proved that free topological groups on infinite connected compact spaces also have the tubby torus as a quotient group.

Theorem 3. [7] Let $F_{G}(X)$ and $A_{G}(X)$ be the Graev free topological group and the Graev free abelian topological group, respectively, on an infinite connected compact Hausdorff space. Then the connected topological groups $F_{G}(X)$ and $A_{G}(X)$ have the tubby torus $\mathbb{T}^{\omega}$ as a quotient group.

It follows from Theorem 2.5 of [3] that every non-metrizable connected locally compact abelian group has the tubby torus as a quotient group. But as a connected locally compact abelian group $G$ is isomorphic as a topological group to the product $\mathbb{R}^{n} \times K$, for some non-negative integer $n$ and compact abelian group $K$, and $\mathbb{R}^{n}$ and all compact metrizable groups are separable, we see that if $G$ is non-separable then it is non-metrizable. So we obtain the following result as a consequence.

Theorem 4. Every non-separable connected locally compact abelian group has the tubby torus as a quotient group.

As mentioned earlier, Problem 1 has been aswered for dual-like groups. In particular there is the following powerful and beautiful theorem.

Theorem 5. [8] If B is the Banach space dual of any infinite-dimensional Banach space, then B has a separable infinite-dimensional quotient Banach space.

Corollary 2. If $B$ is the Banach space dual of any infinite-dimensional Banach space, then $B$ has the tubby torus as a quotient group.

Recall that if $G$ is a (Hausdorff) abelian topological group, then we denote by $\widehat{G}$ the group of all continuous homomorphisms of $G$ into the circle group $\mathbb{T}$, where $\widehat{G}$ has the compact-open topology. 
There is a natural homomorphism $\alpha: G \rightarrow \widehat{\widehat{G}}$. The Pontryagin-van Kampen duality theorem is stated below and a discussion and proof appear in $[9,10]$.

Theorem 6. $[9,10]$ If $G$ is any locally compact abelian group then the map $\alpha$ is an isomorphism of topological groups of $G$ onto $\widehat{\hat{G}}$. Also, if $H$ is a closed subgroup of the locally compact abelian group $G$, then $\widehat{H}$ is a quotient group of $\widehat{G}$, and if $A$ is a quotient group of $G$, then $\widehat{A}$ is isomorphic as a topological group to a closed subgroup of $\widehat{G}$. Further, the map $\alpha$ restricted to $H$ is an isomorphism of topological groups of $H$ onto the subgroup $\alpha(H)$ of $\widehat{\hat{G}}$.

The following is less well-known.

Let $E$ be a locally convex space. As $E$ is a topological group, the topological group $\widehat{E}$ consisting of all continuous group homomorphisms of $E$ into $\mathbb{T}$ with the compact-open topology is a topological group, as is $\widehat{\hat{E}}$. As mentioned above, there is a natural homomorphism of $E$ into $\widehat{\hat{E}}$.

Theorem 7. [11] Proposition 15.2. Let E be a complete metrizable locally convex space (that is a Fréchet space). Then $\alpha$ is an isomorphism of topological groups of $E$ onto $\widehat{\hat{E}}$.

We note that Theorem 7 does not tell us whether, for example $\alpha$ restricted to a closed subgroup $H$ of $E$ is an isomorphism of topological groups of $H$ onto the subgroup $\alpha(H)$ of $\widehat{\hat{E}}$. In fact this is not always true. $\S 11$ of [12] gives an example of a closed subgroup $H$ of a Fréchet space $E$ such that $\alpha$ restricted to $H$ is not an isomorphism of topological groups of $H$ onto its image in $\widehat{\hat{E}}$. To see how badly things can go "wrong", we note Theorem 6.1 of [11]: Let $E$ be a metrizable locally convex space. If $E$ is not a nuclear space, then it has a discrete subgroup $H$ such that there are no non-trivial continuous homomorphisms from $\operatorname{span}(H) / H$ into $\mathbb{T}$, where $\operatorname{span}(H)$ denotes the linear span in $E$ of $H$.

Theorem 5 leads us then to the natural question:

Problem 3. If $E$ is any infinite-dimensional Fréchet space which does not have the weak topology and $\widehat{E}$ is its dual topological group, does $\widehat{E}$ have the tubby torus as a quotient group? In particular, is this the case for $E$ a Banach space or a Schwartz space?

This question is open, however a positive answer is given for nuclear spaces in the next section.

\section{The Main Result}

Definition 1. A topological group $G$ is said to be reflexive if the natural mapping $\alpha$ from $G$ to $\widehat{\hat{G}}$ is an isomorphism of topological groups. The topological group $G$ is said to be strongly reflexive if every closed subgroup and every Hausdorff quotient group of $G$ is reflexive.

Theorem 8. [12] (Theorem 20.35) Every complete metrizable nuclear locally convex space is strongly reflexive.

Proposition 1. [11] (Proposition 17.1(c)) Let $H$ be a closed subgroup of a strongly reflexive topological group G. Then $\widehat{H}$ is isomorphic as a topological group to a quotient group of $\widehat{G}$.

Theorem 9. Let $E$ be a metrizable nuclear locally convex space. Then $\widehat{E}$ has the tubby torus $\mathbb{T}^{\omega}$ as a quotient group if and only if $E$ does not have the weak topology.

Proof. By Theorem 2 of [13], if $H$ is a dense subgroup of the metrizable topogical group $G$, then $\widehat{G}$ is isomorphic as a topological group to $\widehat{H}$. So the dual group $\widehat{E}$ of $E$ is isomorphic as a topological group to the dual group of the completion of $E$. So there is no loss of generality in assuming that $E$ is complete. Further, the completion of a metrizable nuclear locally convex space is a metrizable nuclear locally convex space by Theorems 20.34 and 20.20 of [12]. 
The theorem in [14] says that a locally convex space $E$ has the weak topology if and only if every discrete subgroup of $E$ is finitely generated. However, its proof there gives rather more. Namely, the locally convex space $E$ does not have the weak topology if and only if $E$ contains a discrete free abelian subgroup $S$ which is not finitely generated.

So if the metrizable nuclear locally convex space $E$ does not have the weak topology, then it has a subgroup $S$ isomorphic as a topological group to a restricted direct product of $\mathbb{Z}_{i}, i=1,2, \ldots, n, \ldots$, where each $\mathbb{Z}_{i}$ is isomorphic as a topological group to the discrete $\mathbb{Z}$ of integers. Noting $\S 3$ of [15], we see that the dual group of this restricted direct product of $\mathbb{Z}_{i}$ is the tubby torus $\mathbb{T}^{\omega}$, and it then follows from Theorem 8 and Proposition 1 that $\widehat{E}$ has the tubby torus as a quotient group, as required.

On the other hand if the complete metrizable locally convex space $E$ has the weak topology, then it is isomorphic as a locally convex space to $\mathbb{R}^{\omega}$. So its dual group $\widehat{E}$ is isomorphic as a topological group to the locally convex space $\varphi$. However, as mentioned earlier, it is proved in [5] (and generalized in [7]), that $\varphi$ does not have the tubby torus as a quotient group, which completes the proof.

Funding: This research received no external funding.

Acknowledgments: The author thanks the reviewers for their careful refereeing.

Conflicts of Interest: The author declares no conflict of interest.

\section{References}

1. Ferrando, J.C.; Kakol, J.; López-Pellicer, M.; Śliwa, W. On the separable quotient problem for Banach spaces. Functiones et Approximato Commentarii Mathematici 2018, 59, 153-173. [CrossRef]

2. Morris, S.A.; Yost, D.T. An observation on the separable quotient problem for Banach spaces. Axioms 2020, 9, 7. [CrossRef]

3. Leiderman, A.G.; Morris, S.A.; Tkachenko, M.G. The separable quotient problem for topological groups. Isr. J. Math. 2019, 234, 331-369. [CrossRef]

4. Toruńczyk, H. Characterizing Hilbert space topology. Fundam. Math. 1981, 111, 247-262. [CrossRef]

5. Gabriyelyan, S.S.; Morris, S.A. A topological group observation on the Banach-Mazur separable quotient problem. Topol. Appl. 2019, 259, 283-286. [CrossRef]

6. Eidelheit, M. Zur Theorie der Systeme linearer Gleichungen. Studia Math. 1936, 6, 130-148. [CrossRef]

7. Morris, S.A. A remark on the separable quotient problem for topological groups. Bull. Aust. Math. Soc. 2019, 100, 453-457. [CrossRef]

8. Argyros, S.A.; Dodos, P.; Kanellopoulos, V. Unconditional families in Banach spaces. Math. Ann. 2008, 341, 15-38. [CrossRef]

9. Morris, S.A. Pontryagin Duality and the Structure of Locally Compact Abelian Groups; London Math. Soc. Lecture Notes Series 29; Cambridge University Press: Cambridge, UK, 1977.

10. Hofmann, K.H.; Morris, S.A. The Structure of Compact Groups, 3rd ed.; Walter de Gruyter GmbH: Berlin, Germany, 2013.

11. Banaszczyk, W. Additive Subgroups of Topological Vector Spaces; Springer: Berlin, Germany, 1991.

12. Aussenhofer, L. Contributions to the duality theory of abelian topological groups and to the theory of nuclear groups. In Dissertationes Mathematicae; Polska Akademia Nauk Instytut Matematyczny: Warszawa, Poland, 1999.

13. Chasco, M.J. Pontryagin duality for metrizable groups. Arch. Math. 1998, 70, 22-28. [CrossRef]

14. Morris, S.A. A topological group charachterization of those locally convex spaces having their weak topology. Math. Ann. 1972, 195, 330-331. [CrossRef]

15. Brown, R.; Higgins, P.J.; Morris, S.A. Countable products and sums of lines and circles: Their closed subgroups, quotients and duality properties. Math. Proc. Camb. Philos. Soc. 1975, 78, 19-32. [CrossRef]

(C) 2020 by the author. Licensee MDPI, Basel, Switzerland. This article is an open access article distributed under the terms and conditions of the Creative Commons Attribution (CC BY) license (http://creativecommons.org/licenses/by/4.0/). 